\title{
Perspective of dye-encapsulated conjugated polymer nanoparticles for potential applications
}

\author{
BIKASH JANA ${ }^{1}$ (D, SANTANU BHATTACHARYYA ${ }^{1,2}$ and AMITAVA PATRA ${ }^{1, *}$ (D) \\ ${ }^{1}$ Department of Materials Science, Indian Association for the Cultivation of Science, Jadavpur, Kolkata 32, India \\ ${ }^{2}$ Present address: Department of Chemical Science, Indian Institute of Science Education and Research (IISER), \\ Berhampur, Odisha 760010, India \\ *Author for correspondence (msap@iacs.res.in)
}

MS received 17 January 2018; accepted 1 May 2018; published online 1 September 2018

\begin{abstract}
Design of highly luminescent nanomaterials is an emerging area of research for photonic and bio-photonic applications. Nowadays, dye-encapsulated polymer nanoparticles (PNPs) are found to be very promising alternative nextgeneration luminescent nanomaterials because of extraordinary brightness, easy synthesis, higher photo-stability and nontoxic behaviour. Herein, we have highlighted the dynamics of the fluorophore molecules inside PNPs. Furthermore, we discuss the fundamental correlation of particle brightness with the size of the PNPs as well as population of the dye molecules inside the PNPs. Considering the resonance energy transfer process, generation of white light by varying the dye concentration and singlet oxygen generation using photosensitizer dye have been described. Finally, we discuss the importance of hybrids of conjugated PNPs for potential light harvesting systems such as photovoltaic and optoelectronic applications.
\end{abstract}

Keywords. Polymer nanoparticles; energy transfer; charge transfer; confined motion; artificial light harvesting.

\section{Introduction}

Luminescent nanomaterials have recently garnered considerable attention due to their potential uses in photonics and bio-photonics [1-4]. Consequently, emphasis has been given on designing of dye-encapsulated conjugated polymer nanoparticles (PNPs) as an alternative luminescent source because of several advantages, i.e., easy synthesis procedure, easy to functionalize the surface, higher photo-stability, efficient brightness, broad absorption with extraordinarily high molar extinction coefficient and colour tunability [5-10]. Basically, conjugated polymers are multi-chromophoric systems where delocalization of $\pi$-electrons of every monomeric unit leads to large absorption cross-section [11,12]. Therefore, the optical properties strongly depend on extent of conjugation, electronic delocalization and inter/intramolecular interactions, and the exciton diffusion length is on the order of $5-20 \mathrm{~nm}[13,14]$. By coiling of the polymer molecules with altering of extended state to collapsed state, semiconducting PNPs are formed. The blue- or red-shifting of absorption band of PNPs depends on the nature of the polymer. The blue-shifting of absorption is due to the kinking and bending of polymer backbone [15,16], whereas the red-shifting of absorption occurs due to relatively relaxed and ordered conformations with increased inter-chain interactions $[17,18]$. Pecher and Mecking [19] have described the fundamental photophysics of conjugated PNPs.
Furthermore, the colour tunability and brightness of fluorescent PNPs can be enhanced by encapsulating organic fluorophores inside the PNPs [20-22]. Photophysics of encapsulated dye molecules in PNPs and the confined motion of dye molecules were reported recently [20-23]. The photostability and brightness of dye-encapsulated PNPs are important issues for practical applications. Previous reports have already demonstrated the surface functionalization and their applications in biology and medicine [24-27]. Furthermore, it is evident that the hybrid conjugated PNPs are found to be promising for several optoelectronic devices due to their efficient charge/energy transfer dynamics [28-30]. Therefore, the basic understanding of the photophysics of PNP is important for their further developments.

Considering the emerging field of dye-encapsulated PNPs, we have highlighted a few important issues of dye-doped PNPs in detail. Most importantly, we have addressed the basic photophysical properties of PNP-encapsulated dye molecules and their restricted rotational behaviours inside the heterogeneous PNPs using time resolved spectroscopy to unfold the effect of microenvironment on rotational diffusion of encapsulated dye molecules. Furthermore, the fluorescence correlation spectroscopy (FCS) study helps us to understand the relation of the hydrodynamic diameter and particle brightness with the population of dye molecules inside the particle with varying particle size and dye concentration. Another aspect is the single and multistep energy transfer 
using ultrafast spectroscopy for developing light harvesting systems. Again, we discuss the generation of singlet oxygen by cascade resonance energy transfer from $\pi$-conjugated PNPs-photosensitizer dye conjugates. The designing of light harvesting antenna materials by using self-assembled multichromophoric organic-molecule-encapsulated PNPs has been addressed. The photo-switching and thermoresponsive properties of self-assembled nanostructures and their use as efficient antenna materials are discussed. Fundamental understanding on the photophysics of fluorophore-encapsulated PNPs will pave the way for designing new polymer-based materials for potential applications in optoelectronic, artificial light harvesting and photodynamic therapy.

\section{Synthesis of dye-encapsulated PNPs}

Mainly re-precipitation and mini-emulsion methods are adopted for synthesis of fluorophore-doped polymer nanomaterials (shown in figure 1).

\subsection{Re-precipitation method}

Re-precipitation method is the most common and facile method for synthesis of PNPs in aqueous medium without using any surfactants and electrolytes [23,31]. Initially both the hydrophobic polymer and hydrophobic fluorophore molecules are simultaneously dissolved in an organic solvent (most commonly in tetrahydrofuran $[\mathrm{THF}]$ ) with the help of ultrasonication. The percentage of dye molecules with

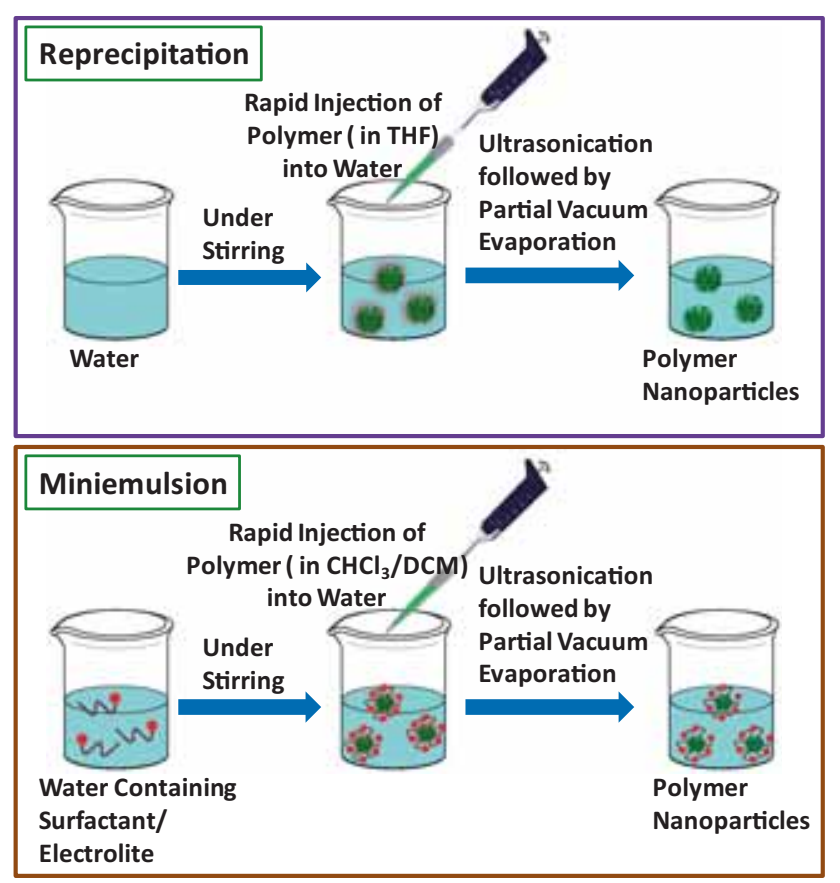

Figure 1. Schematic representation of re-precipitation and miniemulsion techniques for polymer nanomaterials. respect to hydrophobic polymer should be very low $(<5 \mathrm{wt} \%)$ to inhibit the aggregation of fluorophore molecules inside PNPs. Then, the organic solution (containing hydrophobic polymer and fluorescent dye) is rapidly injected into the aqueous medium under vigorous stirring condition. Due to miscibility of organic solvent into polar aqueous medium, hydrophobic polymer and fluorescent dye molecules come in direct contact with the polar medium. To avoid contact with the polar medium, the hydrophobic polymer chain coils up and forms nanoparticle morphology. As fluorescent dye molecules are also hydrophobic in nature, it preferentially enters into the non-polar rigid environment of PNPs. To avoid further swelling of PNPs, partial vacuum evaporation of organic solvent is used. Further, syringe filtration using a micron filter is performed to remove the agglomerated substances. Dialysis is commonly performed by molecular cut-off of 12-14 kD to ensure that all the dye molecules are encapsulated inside the hydrophobic PNPs. Commonly, $>95 \%$ dye molecules remain encapsulated inside the PNPs. The aqueous dispersed dye-doped PNPs remain stable for at least one month. It is known that the surface defect of PNPs appears during the nanoparticle formation, which causes longterm colloidal stability [32]. Negative zeta potential confirms the surface defect formation. Interestingly, modification of the surface charge of PNPs is also possible with the help of long chain aliphatic acid or amine molecules. Most importantly, one can easily tune the size as well as inner matrix rigidity by simply changing the concentration of polymer in stock organic solution and also by changing long alkyl substituent of polymer molecules. This method can also be implanted for one-pot surface functionalization (especially by polystyrene- $\mathrm{COOH}$ derivatives), which is very much important for their further attachment with bio-molecules as well as target-specific attachment in in vitro/vivo bio-imaging devices.

\subsection{Mini-emulsion technique}

Beyond the versatile applicability of re-precipitation technique for the fabrication of dye-doped PNPs, miniemulsion technique is one of the most interesting fabrication procedures [33,34]. In this typical method, initially both the hydrophobic polymers and dye molecules are simultaneously dissolved in an aqueous immiscible organic solvent (like chloroform, dichloromethane or hexane). On the other hand, suitable surfactants are dissolved separately in an aqueous solution and the concentration of the surfactant is carefully maintained (higher with respect to critical micelles concentration). After rapid injection of the organic solvent (containing both polymer and dye) into aqueous medium, ultrasonication leads to formation of many tiny droplets of oil in water. Stable fluorophore-doped PNPs are formed after partial vacuum evaporation of organic solvent. This method can also be useful for the direct polymerization from monomer inside the organic pool in the presence of fluorophore dye molecules. 


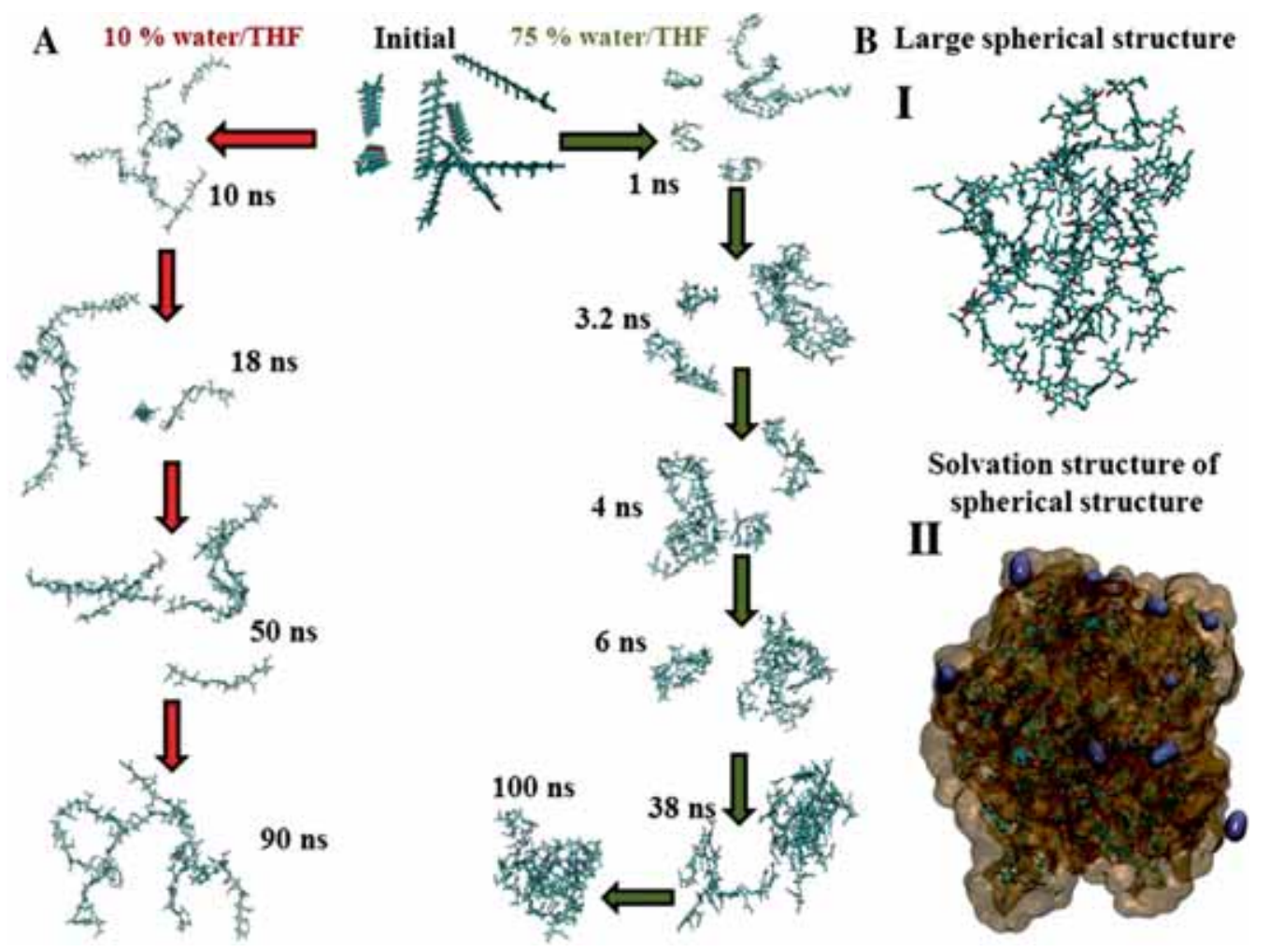

Figure 2. (A) Self-aggregation of the polymer in $10 \%$ and $75 \%(\mathrm{v} / \mathrm{v})$ water-THF mixtures with progression of simulation. The polymer is rendered in cyan CPK mode. (B) (I) The structure of the large spherical selfassembly of the poly[2-methoxy-5-(2-ethylhexyloxy)-1,4-phenylenevinylene] (MEH-PPV) polymer in 75\% $(\mathrm{v} / \mathrm{v})$ water-THF binary mixture obtained after $100 \mathrm{~ns}$ equilibrium simulation is shown. Each of the polymers is rendered as cyan sticks. (II) Solvation structure of the large spherical self-assembly is shown. Polymers are rendered as cyan sticks. THF appears as a brown surface, whereas water is shown in the ice-blue colour. Adapted with permission from ref. [38]. Copyright 2017 American Chemical Society.

Monodispersed NPs formation by controlled fashion is a challenging issue to date.

\section{Formation mechanism of PNPs}

For the synthesis of PNPs by this method, polymers are commonly dissolved in a non-polar solvent (e.g., THF). The polymer molecule in THF solution is in extended form, which is converted to a nanoparticle (collapsed state) with addition of polar solvent (in which hydrophobic polymers are not dissolved, e.g., water). The kinking and bending over polymer backbone is evident to generate collapsed state in PNPs [35-37]. In poly[2-methoxy-5-(2-ethylhexyloxy)-1,4phenylenevinylene] (MEH-PPV) polymer, both theoretical and experimental studies show that a small amount of polar solvent (like water) leads to formation of nanofibres in extended state and with increasing amount of polar solvent, the extended state rapidly collapses and finally leads to NP formation [38]. Recently, we described the formation of different morphologies of polymeric assemblies by molecular dynamic simulation analysis (shown in figure 2). It is shown that $10 \%$ water leads to the start of aggregation of polymer molecules after $\sim 10$ ns and generation of stiff and elongated self-assembled structures after $\sim 18$ ns. Finally, after $90 \mathrm{~ns}$, polymer chains aggregate to form a largely branched fibre-like structure, which matches well with the scanning electron microscope (SEM) image. (The SEM image is presented in ref. [38].) On the addition of $75 \%$ water, nuclei with highly packed core are formed after $\sim 3.2 \mathrm{~ns}$. Small assemblies are merged after $\sim 6 \mathrm{~ns}$ and lead to large spherical particles. The assemblies begin at $\sim 40 \mathrm{~ns}$, which end up with the formation of larger sized assemblies after $\sim 100 \mathrm{~ns}$. Both the concentration of the polymer and the water content dictate the dimension of the spheroid-like structure, which matches with the SEM image. It is noteworthy that the collapsed state again switches to extended state with the addition of non-polar solvent. Photophysical signature of polymeric assembly strongly depends on the morphology. Depending on the nature of polymer, hypsochromic- or bathochromic shift in optical absorption band is found in PNP. However, the red-shifting in photoluminescence (PL) band in PNPs is observed irrespective of the nature of any semiconducting polymer due to energy transfer from high-energy chromophores to low-energy chromophores [15-17,39]. 
Very interestingly, switching in energy migration process is also observed in extended form to collapse form [12,14]. In the extended from, rarely two or few more polymer chains are in the vicinity of each other and inhibit the inter-chain energy migration. Recently, we have seen from time resolved anisotropy study that the intra-chain energy migration (in $790 \mathrm{fs}$ ) is predominant in extended form due to slower depolarization of chromophores and $150 \mathrm{fs}$ fast depolarization is due to inter-chain energy transfer for collapsed state [38].

\section{Dye-encapsulated conjugated PNPs and their photophysics}

Aqueous dispersed dye-doped PNPs with bright luminescence have potential applications in biological imaging [24-26,40,41]. This section will discuss the photophysical behaviours and dynamics of the fluorophore molecules inside PNPs.

\subsection{Confined motion of encapsulated dye molecules inside PNPS}

Interesting findings were reported recently regarding the modified photophysics as well as rigid rotational behaviour of coumarin153 (C153) dye molecules inside different sized poly(9-vinylcarbazole) (PVK) PNP by steady state and time resolved spectroscopy [20]. C153-doped PVK PNPs of three different sizes were fabricated by typical solution-based reprecipitation technique by simply varying the concentration of PVK in stock THF solution, while concentration of C153 remained constant. Efficient energy transfer from the host PVK PNP to the guest C153 dye increases the intensity of PL band of $\mathrm{C} 153$ and a blue-shifting of PL arises due to change of polarity. The relative quantum yield of $\mathrm{C} 153$ dye molecules increases from 0.12 in water to 0.52 in encapsulation inside PNPs. Higher concentration of polymer in THF gives larger size NPs. Therefore, a large number of polymer molecules coiled up together to form a single NP having larger size. As a result, inner matrix rigidity gradually increases with increasing size of the NPs, which directly influences the PL properties of encapsulated dye molecules. It is interesting to note that the decay time is also increased due to encapsulation of dye inside the PNPs (figure 3A). The bi-exponential kinetics of confined dye indicates the heterogeneous inner environments of PNP due to the coiling of the polymer chain. Faster decay component of dye is due to the attachment with more polar caged water inside PNPs, whereas slower decay component is assigned as hydrophobic attachment of dye inside polymer matrix. Interestingly, the enhanced radiative decay rates clearly suggest the enhancement of refractive index. Furthermore, the change in non-radiative decay rates is correlated to the restricted rotation of the dye molecules inside PNPs. The time resolved anisotropy emission decay of C153 inside PNPs is found to be increased and the anisotropy decay follows bi-exponential decay kinetics. The average reorientation time varies from 0.23 to $0.62 \mathrm{~ns}$ on changing the size from 10 to $80 \mathrm{~nm}$ PNPs (figure 3B). Furthermore, the slow and the fast motion are separable in terms of slow lateral diffusion, fast wobbling motion and the overall rotation of the host PNPs. The overall rotation time of PNP is 1000 times larger than even lateral diffusion time of C135 dye. However, the time of lateral diffusion is decreased with increasing size as well as the rigidity of PNPs. The rigidity of PNPs is described through the measurement of order parameter. The magnitude of order parameter is considered to be the spatial restriction of the probe dye molecules and its value varies from 0 to 1 depending upon unrestricted and restricted rotation of dye molecules, respectively. Higher values of the order parameters indicate restricted rotation of dye molecules inside the NPs and it increases with increasing size of the PNPs. Furthermore, the allowed cone angle for encapsulated C153 dye molecules for wobbling motion is directly correlated to the order parameter. The allowed cone angle for encapsulated dye molecules decreases with increasing size of the PVK PNP, indicating that micro-viscosity increases with increasing size of nanoparticles. Analysis reveals that the understanding of the confined motion of encapsulated dye molecules is important for designing efficient systems for potential applications in light harvesting and drug delivery.

\subsection{FCS for particle brightness with number of dye molecules inside PNPS}

Considering the applicability of dye-doped fluorescent PNPs, a few important points need to be addressed: (a) photostability of the NPs, (b) per particle brightness (PPB) and (c) its direct correlation with the size and population of the dopant dye molecules inside the PNPs. The FCS is being used to evaluate the fluorescence intensity fluctuations of fluorescent molecules or fluorescent nanoparticles in an extremely small volume ( $<$ femto litre), which allows one to obtain diffusion coefficient, hydrodynamic radius, particle brightness and concentration. Previously, the FCS study has been used on dye-doped silica NPs [42-44]. The main difference between the silica NP matrix and PNP matrix is the inner environment. The inner environment of PNPs is heterogeneous with different polarities, while silica matrix is commonly homogeneous. The FCS study on Nile red (NR) dye-doped different-sized PVK PNPs using a 532-nm laser source (for the direct excitation of NR dye molecules) is reported [21] and it is found that the hydrodynamic radius is increased from 20 to $55 \mathrm{~nm}$ (shown in figure 3C) on increasing the size of the PNPs from 40 to $100 \mathrm{~nm}$. We have calculated the particle concentration of as-prepared aliquots, which is on the order of $\sim 50-80 \mathrm{nM}$. Again, the average count rate is directly proportional to the photon collected from the same focal volume, which provides the intrinsic PPB of the diffusing particles. Results show that the calculated average PPB is increased with the size of the PNPs and with increasing concentration of dopant dye (figure 3D). Importantly, it is found that dye-doped PNPs are more than one order brighter than what free rhodamine $B$ is in aqueous solution [43]. Number of dye molecules 

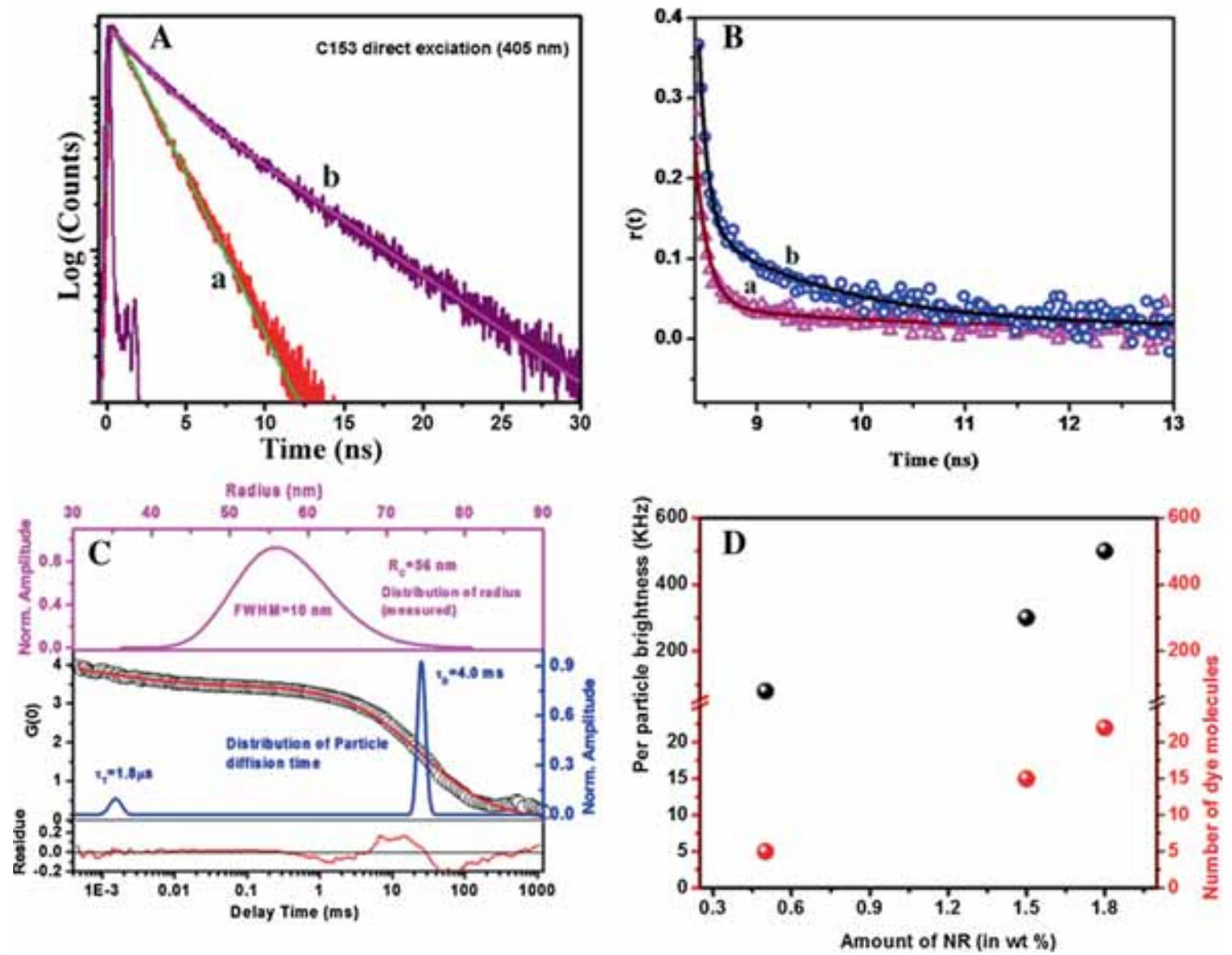

Figure 3. (A) Normalized emission decay of (a) C153 freely dispersed in water and (b) $80 \mathrm{~nm}$ PVK PNP; (B) time resolved anisotropic decay curves of C153 dye encapsulated in (a) $10 \mathrm{~nm}$ and (b) $80 \mathrm{~nm}$ PVK PNPs; adapted with permission from ref. [20]. Copyright 2011 American Chemical Society. (C) Upper panel shows the distribution of hydrodynamic radius, middle panel shows the autocorrelation curves (black circles), the resulting fitting of Maximum-Entropy-Methodbased fitting routine (MEMFCS) (red line), distribution profile of the diffusion time obtained by MEMFCS analysis (blue solid line) and the lower panel shows the residual plot of NR-doped 100 nm PVK PNPs; (D) particle brightness (black ball) and number of dye molecules (red ball) with varying concentration of the dye molecules; adapted with permission from ref. [21]. Copyright 2013 American Chemical Society.

per PNP eventually influences the PL properties of encapsulated dye molecules inside polymer matrix. The calculated dye molecules per particle are found to be $\sim 5, \sim 15$ and $\sim 22$ for $0.5,1.5$ and $1.8 \mathrm{wt} \%$ NR-doped PVK PNPs (size $\sim 100 \mathrm{~nm}$ ), respectively. Time resolved emission decay analysis suggests that the rigidity increases with increasing size of the PNP, which enhances the restricted rotation of the dye molecules. It eventually alters the particle brightness. In addition, the heterogeneous distributions of the dye molecules inside PNP increase with increasing population of the dye molecules and enhances the possibility of energy hopping between different NR dye molecules.

\section{Host-guest energy transfer process}

Very often, low-energy-gap dopants are blended/doped with a high-band-gap host to enhance the efficiency of organic light-emitting diode (OLED) [5,45]. Doping also helps diminish the concentration quenching of the dopant due to large stokes shift and tune the emission colour with increasing lifetime of the devices. During performance of OLED, electrons and holes are injected from opposite electrodes to generate excitons and these excitons are harvested by the devices [46]. Here, we discuss various types of dye-doped PNPs for tuning the light emission and to increase the energy transfer efficiency.

\subsection{Single dye-encapsulated system}

In dye-doped conjugated PNPs, host conjugated polymer acts as an efficient antenna material to harvest light energy due to large absorption cross-section and efficient energy transfer from polymer to the encapsulated dye molecules. FRET-based energy transfer defines an additional deactivation pathway for the excited donor moiety. The criterion for effective energy 

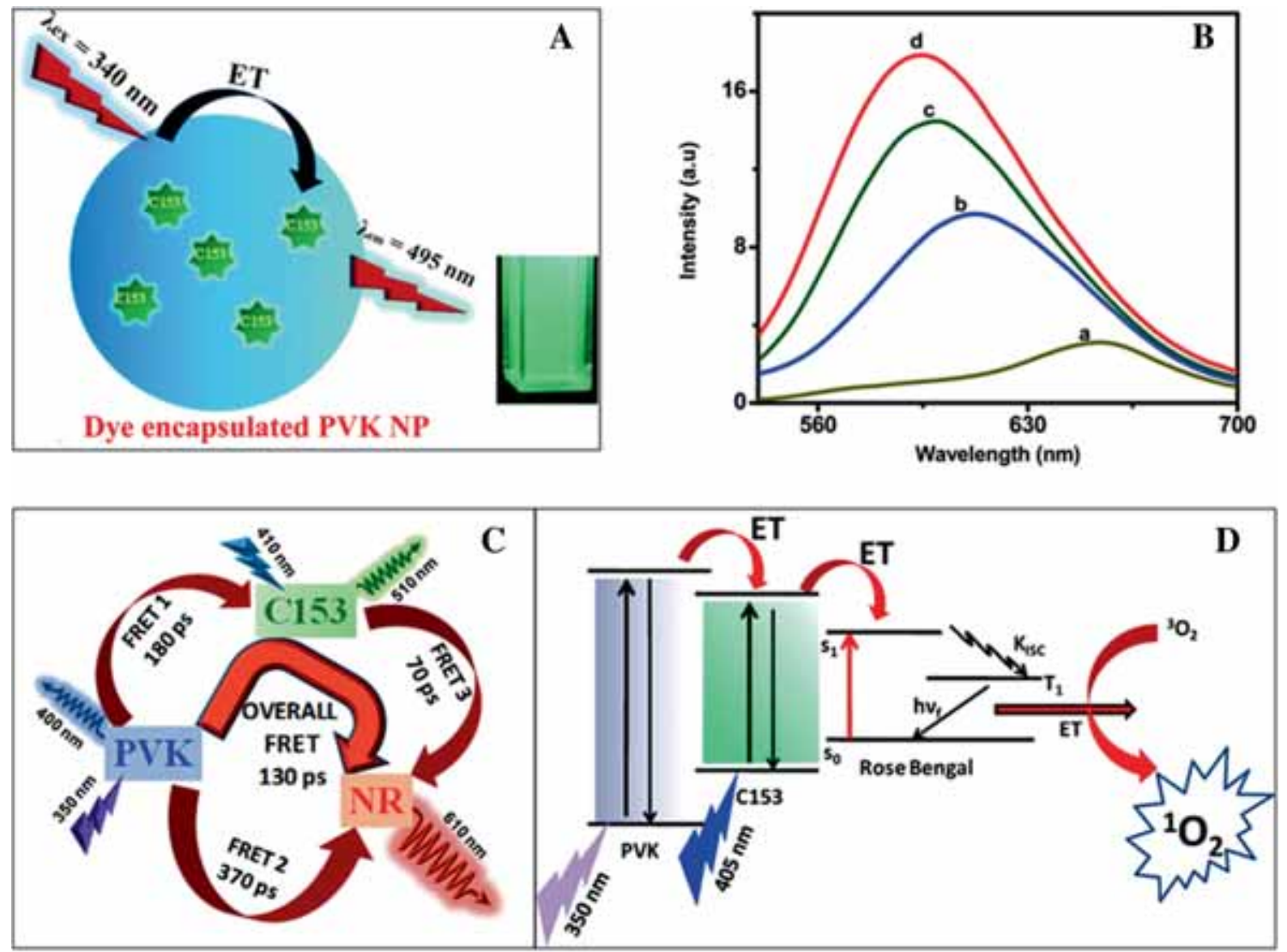

Figure 4. (A) Schematic representation for the energy transfer from PVK host to C153 dopant dye; adapted with permission from ref. [20]. Copyright 2011 American Chemical Society. (B) Enhancement in photoluminescence of NR from (a) water and NR-doped (b) $40 \mathrm{~nm}$, (c) $60 \mathrm{~nm}$ and (d) $100 \mathrm{~nm}$ PVK PNPs. Adapted with permission from ref. [21]. Copyright 2013 American Chemical Society. (C) Schematic representation showing the different energy transfer rates in dual dye (C153 and NR) PVK PNP; adapted with permission from ref. [22]. Copyright 2014 Royal Society of Chemistry. (D) Schematic representation of singlet oxygen generation based on cascade energy transfer from PVK to RB via C153; adapted with permission from ref. [51]. Copyright 2014 American Chemical Society.

transfer is the large overlap between the emission spectrum of donor and the absorption spectrum of acceptor. Besides dipole-dipole approximation, line-dipole approximation is considered in polymer-based energy transfer $[9,47]$. In addition, the distance between donor and acceptor also dictates the efficiency of energy transfer. Generally, movement of localized excitons from one site to the other in polymeric materials occurs through spontaneous hopping by FRET or Dexter process. However, coherent inter-chain electronic energy transfer is found among delocalized excitons at cryogenic temperatures and even at room temperature in the first $100 \mathrm{fs}$ after excitation $[12,48,49]$. Most importantly, the exciton diffusion and intra-/inter-exciton transfer process throughout the conjugated polymer strongly influence energy transfer from host polymer to guest dye molecules. Generally, the energy transfer in dye-doped PNP system is investigated by monitoring the quenching of emission of the host polymer and the enhancement in acceptor (dopant) emission with varying concentration of dopant dye molecules. In most cases, multiple polymer chains having multiple chromophoric units are quenched by a single dye molecule [5,7]. It has been reported that efficient energy transfer process occurs from PNPs to encapsulated C153 dye and NR dye molecules separately $[20,21]$. Both steady state and time resolved studies confirm the excited state interaction between the host (PNP) and guest (C153). Steady state PL spectroscopy depicts almost $100 \%$ quenching for PVK at donor excitation and a broad blue-shifted enhanced peak appears from encapsulated C153 dye molecules. It indicates the efficient energy transfer from host PVK to dopant C153 dye molecules (figure 4A). With varying concentration of dopant dye molecules inside PVK PNPs, the extent of PL quenching of host molecules can be estimated using the following Stern-Volmer relation [50]:

$$
F_{0} / F=1+K_{\mathrm{sv}}[\mathrm{A}] .
$$

Here, $F_{0}$ and $F$ are fluorescence intensities due to PVK in the absence and presence of dye, respectively. $K_{\mathrm{sv}}$ defines 
the Stern-Volmer quenching constant and [A] indicates the concentration of the acceptor molecules. The Stern-Volmer plot suggests that about 11 polymer molecules are quenched by a single C153 dye molecule [20]. Further data obtained from time resolved spectroscopy indicate the drastic shortening decay time of PVK (from 1.10 to $0.1 \mathrm{~ns}$ ) in the presence of C153 dye molecules, suggesting the efficient energy transfer (>90\%) from host PVK to guest dye molecules. Recently, the efficient energy transfer from PVK to NR dye was confirmed by the steady state (figure 4B) and time resolved spectroscopic measurements [21]. Enhancement of fluorescence intensity and the excited state lifetime of NR with the excitation of donor PNPs further confirms the energy transfer. It is reported that the brightness increases with increasing size of host PNP. Furthermore, photo-stability of the encapsulated dye is investigated by measuring the fluorescence intensity of the acceptor dye molecules. Analysis reveals that efficient energy transfer and large enhancement in photo-stability of NR-encapsulated PVK are observed.

\subsection{Multiple dye-encapsulated systems}

With an advancement of single-fluorophore-doped systems, the cascade energy transfer of semiconducting PNPs doped with multiple dyes is also designed to tune the emission with enhanced quantum yield. As already explained, the energy transfer from PVK to both C153 and NR is significant to produce only one emission colour. PVK PNPs doped with both green-emitting C153 and red-emitting NR dye molecules have been designed to produce tunable emission colour with varying dye concentration. The possible energy transfer pathways are: (a) direct energy transfer from host PVK to C153, (b) direct energy transfer process from host PVK to NR, (c) FRET between two dopant dye molecules (C153 to NR) inside polymer nanodomain and finally (d) by the cascade energy transfer process from PVK to NR dye molecules through C153 [22]. Steady-state PL spectroscopy is undertaken to understand the photophysical processes for dual-dye-doped PVK PNPs. At a fixed concentration of C153, the PL quenchings of PVK PNPs and C153 are observed with gradually increasing concentration of NR. On the other hand, when the content of C153 is varied at fixed NR concentration, the PL intensities of both C153 and NR are increased. With simultaneous co-doping of C153 and NR, PVK PNPs transfer their energy to NR through C153 because of better spectral overlap between PVK and NR via $\mathrm{C} 153$ dye, resulting in a cascaded ET process: PVK to $\mathrm{C} 153$ to NR. Therefore, the $\mathrm{C} 153$ molecules act as a molecular ladder, which enhances the ET efficiency from the excited PVK host to the trapped NR. For detailed investigations on the rate of energy transfer process in each and individual step, acceptor rise time data analysis is performed upon exciting the sample with a femtosecond (fs) laser pulse for excitation wavelength of three chromophores. In the PVK:C153 system, the observed rise time of 180 ps confirms the energy transfer from PVK to C153, which completely vanishes in the PVK:C153:NR system. A rising component of 370 ps for NR dye molecules in PVK:NR system also ensures the energy transfer from PVK to NR. However, the very fast 70 ps rise time for C153:NR system (at the excitation of C153) arises due to the close proximity between the two dyes and their spectral overlap. In presence of $\mathrm{C} 153$ after excitation of PVK PNP, the observed rise time is 130 ps in NR emission, which confirms the cascade energy transfer. Analysis suggests $180 \mathrm{ps}$ rise time for the energy transfer from PVK to C153, 370 ps for the energy transfer from PVK to NR, 70 ps for energy transfer from $\mathrm{C} 153$ to $\mathrm{NR}$ and 130 ps for the global energy transfer process from PVK to C153 to NR (shown in figure 4C). The tunable emission from blue to red to bright white light generation is achieved by manipulating the dopant concentrations [22]. The multistep energy transfer process is being used for generation of singlet oxygen $\left({ }^{1} \mathrm{O}_{2}\right)$ by the employment of C153-doped PVK PNPs with surface-attached Rose Bengal (RB) dye molecules (figure 4D) [51]. Negatively charged RB dye molecules are attached to the surface-modified positively charged PNP through electrostatic attachment. Steady-state and time resolved data confirm the cascaded energy transfer from PVK PNP to surface-attached RB dye molecules through encapsulated dye C153 molecules. The intersystem crossing from lower excited singlet state to triplet state enhances the transformation of triplet oxygen $\left({ }^{3} \mathrm{O}_{2}\right)$ to singlet oxygen $\left({ }^{1} \mathrm{O}_{2}\right)$ in solution medium. The PL peak at $1270 \mathrm{~nm}$ confirms the generation of singlet oxygen. Quantum yield of singlet oxygen is verified by photo-degradation of 2-Chlorophenol in $\mathrm{D}_{2} \mathrm{O}$ medium. Thus, dye-doped PNPs could be employed as efficient antenna materials to generate photo-stable bright tunable emission and singlet oxygen for photodynamic therapy.

\section{Self-assembled molecules for efficient antenna materials in artificial light harvesting}

In natural photosynthesis, antenna units composed of appropriately organized large number of chlorophyll molecules absorb the solar light and then the absorbed light energy is funnelled towards reaction centre via a very fast and efficient cascade and homo energy transfer process. Finally, the charge separation via electron transfer leads to storage of solar energy as chemical energy. To mimic the photosynthesis process, the following challenges are taken to construct an efficient artificial light harvesting system: (a) appropriate well-defined organized arrangements of chromophores, (b) fast and efficient energy transfer between chromophores and (c) charge transfer to generate long-lived charge-separated state. This section discusses these processes to design artificial light harvesting systems.

Self-assembled conjugated multi-chromophoric organic nanostructured materials have been used for developing efficient light harvesting materials because the electronic properties of the excitons can be modified by packing of molecules. Among aggregates, $\mathrm{H}$-aggregate nanostructures are more 

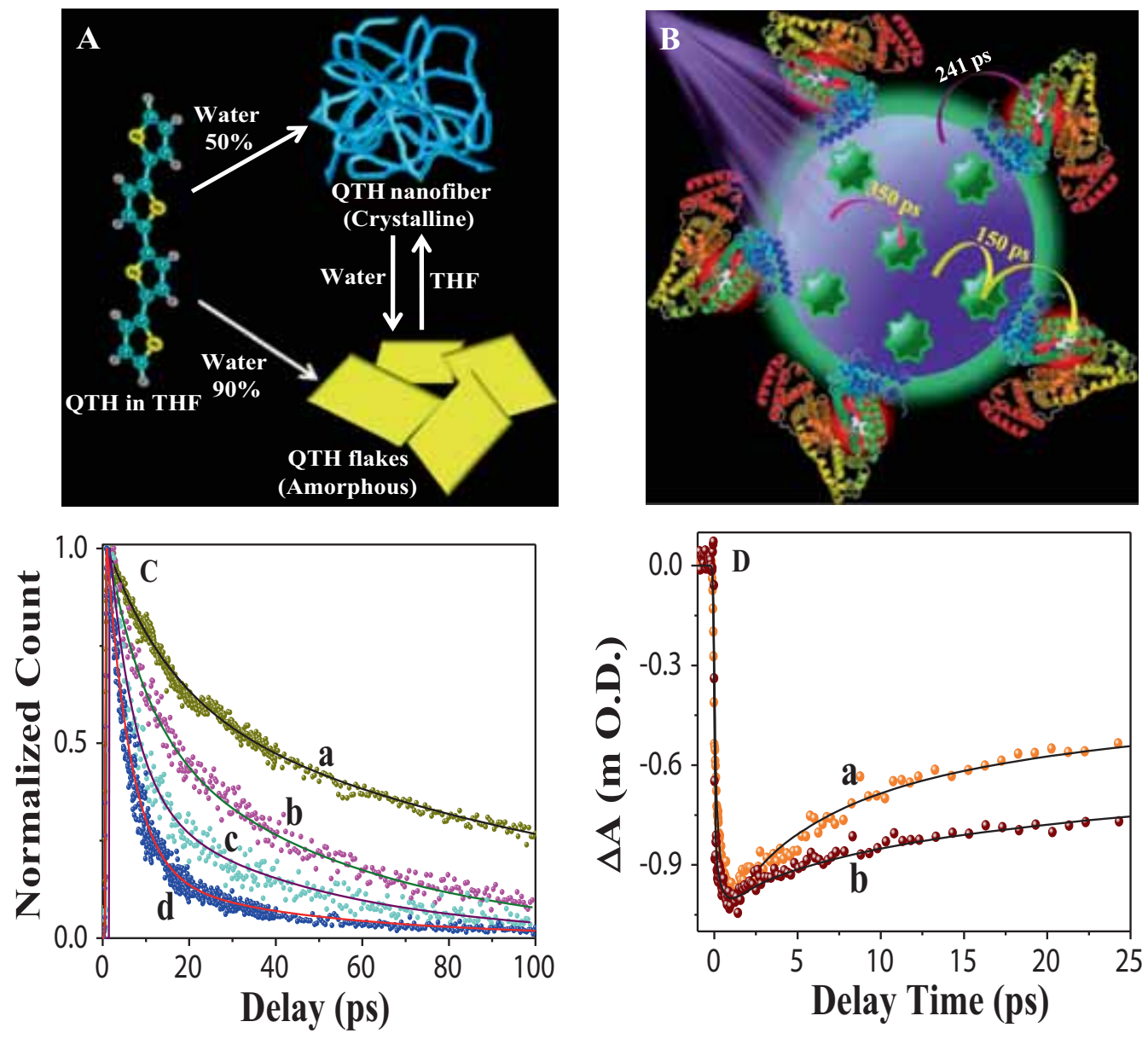

Figure 5. (A) Schematic representation of reversible transformation of QTH fibre and flakes nanostructures; adapted with permission from ref. [54]. Copyright 2015 John Wiley \& Sons, Inc. (B) Schematic representation of the energy transfer pathways in a C153-doped PVK PNP-BSA system; reproduced from ref. [56] with permission from Royal Society of Chemistry. (C) Femtosecond fluorescence decays of pure P3HT PNP with increasing Au NP concentration [(a) $0 \mathrm{nM}$, (b) $4.7 \mathrm{nM}$, (c) $6.3 \mathrm{nM}$ and (d) $7.8 \mathrm{nM}$ of Au NP]; reproduced from ref. [57] with permission from the PCCP Owner Society. (D) Bleach recovery kinetics of (a) large-sized CdTe QDs and (b) hybrid; adapted with permission from ref. [61]. Copyright 2016 American Chemical Society.

suitable because they have indirect excitons, which increase the excited state lifetime, and excitation in $\mathrm{H}$-aggregates leads to production of delocalized singlet excitons at room temperature [52,53]. Furthermore, extent of H-aggregation in quarterthiophene (QTH) is controlled by changing the solvent polarity [54]. With increasing solvent polarity, the morphology of the aggregated nanostructure is changed from fibre to flake with decreasing crystallinity. Moreover, the microstructures are not rigid enough with any morphology and thus photo-switching and thermoresponsive PL properties are found in both nano/microstructures (figure 5A). The relaxation dynamics reveals that gradual increment of the excited state of aggregated domain corresponds to the enhancement of H-aggregation from fibre- to flake-type morphology, which is promising for photo-driven devices. Based on this promising aggregated nanostructures, QTH molecules are assembled inside PNPs to be employed as efficient antenna materials for energy transfer towards NR dye inside nanodomains [55]. Spectroscopic investigations show an efficient energy transfer with 10 ps rise time in acceptor (NR) emission with $57 \%$ energy transfer efficiency. As a measurement of light harvesting efficiency, antenna effect of this system is found to be 11.94 with donor to acceptor ratio of 0.008 . Moreover, reaching to more efficient light harvesting, a system has been designed where light energy is transferred inside NR-dye-encapsulated protein via intermediate chromophores through multistep cascade energy transfer (figure 5B) [56]. Here, C153-doped PVK PNP is employed as an antenna material due to its large absorption cross-section, which is promoted by doping of the fluorescent dye molecules inside 
the PNPs. On insertion of intermediate chromophores, C153 enhances the antenna effect from 28 to 31 with donor to acceptor ratio of 0.82 . Taking the advantage of efficient energy transfer, tunable emissions with white light emission have been generated for potential applications in optoelectronic devices.

Like energy transfer, another important aspect of light harvesting process is the charge transfer, which improves the power conversion efficiency (PCE) of the photovoltaic device. Organic-inorganic hybrid materials are the most discussed materials for efficient charge transfer. There are two main ways in which charge transfer process occurs: one is electron transfer at the interface originating from the photoexcited organic counterpart to the inorganic counterpart and second one is hole transfer process from the photo-excited inorganic part to its hole-accepting organic part. Electron transfer is probed in a hybrid material composed of poly(9,9dioctylfluorene-2,7-diyl-co-benzothiadiazole) (P3HT) PNP electrostatically attached to electron-accepting materials, gold (Au) NP [57]. Photo-excitation leads to a gradual bathochromic shift along with the enhancement of absorption bands of P3HT PNPs with the attachment of Au NPs. Shortening of faster part of excited stated lifetime of P3HT PNP with addition of Au NP (figure 5C) and the band alignment of P3HT and $\mathrm{Au}$ NP indicate the electron transfer process. As a proof of this electron transfer process, photocatalysis is improved from 55\% for pure P3HT PNPs to $90.6 \%$ for P3HT PNPs-Au NPs hybrid system under visible light irradiation. Considering the high electron-accepting property of reduced graphene oxide (r-GO), MEH-PPV PNP/r-GO has been designed for more efficient electron transfer [58]. The appearance of broad bleach centred at $635 \mathrm{~nm}$ in transient spectroscopic study confirms the electron transfer from PNP to r-GO. Finally, prompt electron transportation from PNPs to r-GO leads to 2.6-fold enhancement of photocurrent in composite materials under visible light illumination. Importantly, the hole transfer can be steered by changing the distance between the donor and acceptor and the energy level offset between the highest occupied molecular orbital (HOMO) levels of donor and acceptor to generate free charge pairs overcoming the coulombic attraction. A hybrid system composed of $\alpha$-sexithiophene (STH) NP and water-soluble cadmium telluride (CdTe) QDs has been designed for efficient hole transfer [59] process. Based on quenching in emission intensity of QDs in the presence of STH NPs and offset in valence band of QDs $(-6.3 \mathrm{eV})$ and HOMO of STH $(\sim-5 \mathrm{eV})$, the hole transfer process from the QDs to the STH NPs is confirmed. A positive surfactant, cetrimonium bromide (CTAB), is used for the synthesis of STH NPs, which decreases the hole transfer rate due to long intermediate spacer CTAB. To overcome this obstacle, a bi-colour organic fluorophore material [rhodamine 6G] [ monoionic fluorescein] ([R6G][HFL]) has been attached to the negatively charged PVK PNPs through electrostatic attachment to bring donor and acceptor closer to each other [60]. This reduced distance and type II alignment of PVK and [R6G][HFL] lead to hole transfer from dye to PVK with a relatively faster time scale. To achieve better efficiency in photo-current generation for the hybrid system, the hole transfer rate also needs to be tuned. Very recently, modulation of hole transfer was performed by tuning the energy level of the inorganic counterpart through the size variation of QDs in $\mathrm{CdTe}-$ MEHPPV hybrid materials [61]. Depending on the valence band offset of QDs and PNP, the faster hole transfer rate is found in small-sized QDs. However, with increasing size of QDs, the valence band offset is decreased, which leads to more sluggish bleach recovery kinetics of QDs in the presence of PNPs (figure 5D). Thus, fine-tuning of both electron and hole transfer rates leads to improved photovoltaic devices.

\section{Conclusion and outlook}

Important issues like brightness, photo-stability and dynamics of dye inside PNP, and energy transfer for artificial light harvesting are discussed. Dye-doped conjugated PNPs are now well recognized for alternative next-generation luminescence nanomaterials due to extraordinary brightness, easy synthesis, higher photo-stability and nontoxic behaviour. Extraordinary brightness and photo-stability of dye-doped conjugated PNP are being used for both in-vivo and invitro bio-imaging applications. In addition, fluorescent PNPs have been found to be potential candidates for clinical cancer detection, drug delivery and gene delivery therapeutics devices. In addition, the FRET-based photosensitization as well as single-oxygen generation capability also increases the promises of dye-doped PNPs in photodynamic therapy for oncology and cancer treatment. This is in embryonic stage; more research is required to open up new possibilities in near future. Tunable PL and white light generation from multipledye-doped PVK PNP both in solution and solid state would be promising for light-emitting devices. Further research on multiple-dye-doped PNPs will definitely open up new prospects for photovoltaic devices and OLED devices. The ordered arrangement of dye molecules inside polymer nanofibres may enhance the applicability of laser emission and other photo-driven devices. Study of the inter-/intra-chain energy transfer of the self-assembled/aggregated nanostructures by ultrafast spectroscopy is essential for developing efficient devices. Moreover, polymer-based organic-inorganic hybrids are the most efficient materials for improving quantum efficiency in solar-cell-based applications. The understanding of charge separation in inorganic-organic hybrid systems is very important to design efficient devices for light harvesting applications. Other important issues in optoelectronic applications are exciton diffusion and diffusion length, which are not well understood in conjugated PNPs. Ultrafast spectroscopic study is very important to address these important issues. Therefore, the fundamental photophysical properties of dye-doped polymer and hybrids composed of polymers will open up new possibilities for future development of photonics, biophotonics and optoelectronic devices. 


\section{Acknowledgements}

'DST-TRC' is gratefully acknowledged for financial support. BJ thanks CSIR for awarding fellowship.

\section{References}

[1] Croce R and van Amerongen H 2014 Nat. Chem. Biol. 10492

[2] Armaroli N and Balzani V 2007 Angew. Chem. Int. Ed. 4652

[3] Barber J 2009 Chem. Soc. Rev. 38185

[4] Lewis N S and Nocera D G 2006 Proc. Natl. Acad. Sci. USA 103 15729

[5] Jana B, Ghosh A and Patra A 2017 J. Phys. Chem. Lett. 84608

[6] Kundu S and Patra A 2017 Chem. Rev. 117712

[7] Bhattacharyya S and Patra A 2014 J. Photochem. Photobiol. C: Photochem. Rev. 2051

[8] Brabec C J 2004 Sol. Energ. Mat. Sol. Cells 83273

[9] Hedley G J, Ruseckas A and Samuel I D W 2017 Chem. Rev. 117796

[10] Barbara P F, Gesquiere A J, Park S-J and Lee Y J 2005 Acc. Chem. Res. 38602

[11] Hwang I and Scholes G D 2011 Chem. Mater. 23610

[12] Collini E and Scholes G D 2009 Science 323369

[13] Scholes G D and Rumbles G 2006 Nat. Mater. 5683

[14] Beljonne D, Pourtois G, Silva C, Hennebicq E, Herz L M, Friend R H et al 2002 Proc. Natl. Acad. Sci. USA 9910982

[15] Padmanaban G and Ramakrishnan S 2000 J. Am. Chem. Soc. 1222244

[16] Klaerner G and Miller R D 1998 Macromolecules 312007

[17] Wang F, Han M-Y, Mya K Y, Wang Y and Lai Y-H 2005 J. Am. Chem. Soc. 12710350

[18] Ong B S, Wu Y, Liu P and Gardner S 2005 Adv. Mater. 171141

[19] Pecher J and Mecking S 2010 Chem. Rev. 1106260

[20] Bhattacharyya S, Paramanik B and Patra A 2011 J. Phys. Chem. C 11520832

[21] Bhattacharyya S, Prashanthi S, Bangal P R and Patra A 2013 J. Phys. Chem. C 11726750

[22] Martin C, Bhattacharyya S, Patra A and Douhal A 2014 Photochem. Photobiol. Sci. 131241

[23] Kurokawa N, Yoshikawa H, Hirota N, Hyodo K and Masuhara H 2004 ChemPhysChem 51609

[24] Hubbell J A and Chilkoti A 2012 Science 337303

[25] Jin Y, Ye F, Zeigler M, Wu C and Chiu D T 2011 ACS Nano 5 1468

[26] Wu W C, Chen C Y, Tian Y, Jang S H, Hong Y, Liu Y et al 2010 Adv. Funct. Mater. 201413

[27] Tuncel D and Demir H V 2010 Nanoscale 2484

[28] Tang C W, VanSlyke S A and Chen C H 1989 J. Appl. Phys. 65 3610

[29] Gather M C, Köhnen A and Meerholz K 2011 Adv. Mater. 23 233

[30] Park E J, Erdem T, Ibrahimova V, Nizamoglu S, Demir H V and Tuncel D 2011 ACS Nano 52483

[31] Wu C, Szymanski C and McNeill J 2006 Langmuir 222956

[32] Clafton S N, Beattie D A, Mierczynska-Vasilev A, Acres R G, Morgan A C and Kee T W 2010 Langmuir 2617785
[33] Kietzke T, Neher D, Landfester K, Montenegro R, Guntner R and Scherf U 2003 Nat. Mater. 2408

[34] Kietzke T, Neher D, Kumke M, Montenegro R, Landfester K and Scherf U 2004 Macromolecules 374882

[35] Saikin S K, Eisfeld A, Valleau S and Aspuru-Guzik A 2013 Nanophotonics 221

[36] Bodunov E N, Berberan-Santos M N and Martinho J M G 2001 Chem. Phys. Lett. 340137

[37] Burlatsky S F, Oshanin G S and Mogutov A V 1990 Phys. Rev. Lett. $\mathbf{6 5} 3205$

[38] Ghosh A, Jana B, Chakraborty S, Maiti S, Jana B, Ghosh H N et al 2017 J. Phys. Chem. C 12121062

[39] Shimizu H, Yamada M, Wada R and Okabe M 2007 Polym. J. 4033

[40] Sarovar M, Ishizaki A, Fleming G R and Whaley K B 2010 Nat. Phys. 6462

[41] Fleming G R, Schlau-Cohen G S, Amarnath K and Zaks J 2012 Faraday Discuss. 15527

[42] Burns A, Ow H and Wiesner U 2006 Chem. Soc. Rev. 351028

[43] Larson D R, Ow H, Vishwasrao H D, Heikal A A, Wiesner U and Webb W W 2008 Chem. Mater. 202677

[44] Ow H, Larson D R, Srivastava M, Baird B A, Webb W W and Wiesner U 2005 Nano Lett. 5113

[45] Laquai F, Park Y-S, Kim J-J and Basché T 2009 Macromol. Rapid Commun. 301203

[46] Burroughes J H, Bradley D D C, Brown A R, Marks R N, Mackay K, Friend R H et al 1990 Nature 347539

[47] Denis J-C, Schumacher S, Hedley G J, Ruseckas A, Morawska P O, Wang Y et al 2015 J. Phys. Chem. C 119 9734

[48] Dubin F, Melet R, Barisien T, Grousson R, Legrand L, Schott M et al 2005 Nat. Phys. 232

[49] Dykstra T E, Hennebicq E, Beljonne D, Gierschner J, Claudio G, Bittner E R et al 2009 J. Phys. Chem. B 113656

[50] Lakowicz J R 2006 Principles of Fluorescence Spectroscopy (New York: Springer)

[51] Bhattacharyya S, Barman M K, Baidya A and Patra A 2014 J. Phys. Chem. C 1189733

[52] Chaudhuri D, Li D, Che Y, Shafran E, Gerton J M, Zang L et al 2011 Nano Lett. 11488

[53] Haedler A T, Kreger K, Issac A, Wittmann B, Kivala M, Hammer $\mathrm{N}$ et al 2015 Nature $\mathbf{5 2 3} 196$

[54] Bhattacharyya S, Jana B, Sain S, Barman M K, Pradhan S K and Patra A 2015 Small 116317

[55] Bhattacharyya S, Jana B and Patra A 2015 ChemPhysChem 16 796

[56] Jana B, Bhattacharyya S and Patra A 2016 Nanoscale 816034

[57] Jana B, Bhattacharyya S and Patra A 2015 Phys. Chem. Chem. Phys. 1715392

[58] Ghosh A, Jana B, Maiti S, Bera R, Ghosh H N and Patra A 2017 ChemPhysChem 181308

[59] Bhattacharyya S, Paramanik B, Kundu S and Patra A 2012 ChemPhysChem 134155

[60] Das S, Jana B, Debnath T, Ghoshal A, Das A K and Patra A 2017 J. Phys. Chem. C 1214050

[61] Jana B, Ghosh A, Maiti S, Bain D, Banerjee S, Ghosh H N et al 2016 J. Phys. Chem. C 12025142 